\title{
Sex and age impact prevalence and symptoms of vasovagal syncope: Implications for accurate diagnosis
}

\author{
Hailey Gregson ${ }^{1}$ and Ana Ivkov² \\ ${ }^{1}$ Crescent Heights High School, Alberta, Canada \\ 2 Department of Psychology, University of British Columbia, British Columbia, Canada \\ KEYWORDS: vasovagal syncope, sex, age, symptoms, misdiagnosis
}

\section{INTRODUCTION}

Syncope is characterized by the transient loss of consciousness followed by spontaneous recovery. The mechanism which underlies this condition is reduced blood flow to the brain [1]. Vasovagal syncope, often termed reflex syncope, is the most common type of syncope [1]. Vasovagal Syncope is caused by the abnormal autonomic reflex to certain stimuli such as pain, micturition/defecation, fear, seeing blood, etc., which results in vasodilation and often times, bradycardia [1].

$3 \%$ of patient cases in hospital emergency departments are caused by syncope, with $1 \%$ of cases resulting in hospital admission [2]. Syncope negatively impacts an individual's quality-of-life, including physical health, mental health, and the ability to perform normal daily activities. The effective diagnosis and management of this condition can help increase a patient's standard of living [3].

Vasovagal syncope is commonly misdiagnosed when patient characteristics are not taken into proper consideration. Two important diagnostic factors include patient age and sex. Patients within these categories show different prevalence's of syncope and present with distinctive symptoms. An incorrect diagnosis may result if the varying symptoms associated with a patient's sex and age are not well-understood and considered during the diagnostic process. To ensure patients are diagnosed correctly, an effort should be made to properly understand and consider these distinctions.

\section{PATHOPHYSIOLOGY OF SYNCOPE}

Vasovagal syncope is often caused by specific actions, such as standing too long, micturition, or a frightening event. The pathophysiology of vasovagal syncope is complex. In brief, certain proactive situations will cause the vagus nerve to function abnormally, leading to vasodilation and/ or bradycardia which causes a significant reduction in blood pressure and low brain perfusion, resulting in syncope.

Vasovagal syncope is often associated with prodromal symptoms. These are early symptoms that signal illness and occur before the onset of transient loss of consciousness (TLOC). Prodromal symptoms include dizziness, pallor, diaphoresis, nausea, and needing to lie down. The prevalence of these symptoms differs by age and sex.

\section{DIAGNOSIS AND MISDIAGNOSIS OF VASOVAGAL SYNCOPE}

The diagnosis of vasovagal syncope is often made clinically, and testing is usually not required. In selected patients, headup tilt table testing is a method used to induce syncope in a controlled environment. Excess venous pooling in lower body veins caused by tilting results in reduced blood return to the heart which can trigger a vagal response [6]. The head-up tilt table test therefore mimics orthostatic stress, a common stress in humans that pulls blood away from the heart and results in excess pooling of blood in the legs, which provokes vasovagal syncope and associated symptoms [6]. Despite its prevalence, syncope can often be mistaken for other conditions or overlooked entirely. It is critical to ensure an accurate diagnosis since an incorrect diagnosis can lead to an inappropriate treatment plan [7]. It is essential to understand the variation of incidence and clinical features of reflex syncope by age and sex, as these demographic features are key for an accurate diagnosis [8].

\section{SYMPTOMS VARY WITH AGE AND SEX}

Causes of vasovagal syncope are known to vary by age and sex. Vasovagal syncope is the most common syncope of all age groups; however, cardiac causes of syncope increase in older patients. Furthermore, patient sex becomes a key 
distinguishing factor for patients in younger age groups.

To study the prevalence of common vasovagal syncope symptoms in patients based on age and sex, Romme et al., divided patients into three age groups: below 40 years (young adults), 40-59 years (middle-aged adults), and 60 years or above (older adults) [7]. The age groups showed different rates of various types of syncope. Reflex syncope was the most common overall, however, its incidence rate was higher in the youngest age group. Comparatively, the oldest age group showed higher rates of cardiac syncope than the youngest age group. Prodromal symptoms were less prevalent in older patients; this may be the result of decreased autonomic nervous system activity and organ function with age [7]. While patients less than 40 years old experienced varying symptoms, the majority of patients above 59 consistently experienced pallor, or extreme paleness (Figure 1).

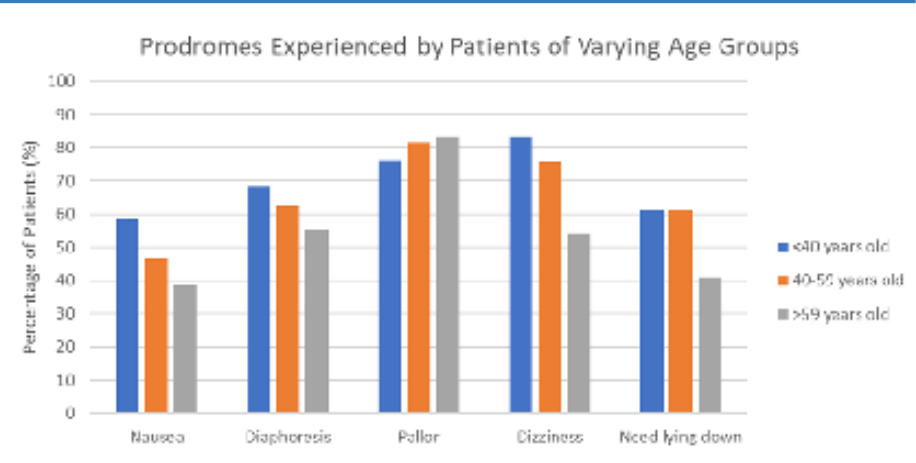

Figure 1. The large differences between prodromes experienced in various age groups. Figure produced with data from [7].

In this study, Romme et al., used broad age categories which raises the issue that some distinctions of syncope symptom prevalence among age groups may still be undiscovered. Further studies specifically investigating the older age group have been conducted. Unger et al., studied adults aged 65 or older and found that patients within the older age category experienced even more distinct prodromal symptoms than previously reported. Those between the ages of 65-74 experienced more prodromal symptoms while in the supine position, whereas patients above the age of 75 experienced more prodromal symptoms while standing [8]. This indicates that syncope is experienced differently among older patients at specific ages. While these studies focus on age, the sex of a patient is also an important determining factor when diagnosing syncope. Within younger patients, syncope is much more prevalent among females than males [9].

\section{VASOVAGAL SYNCOPE AND FEMALE SEX HORMONES}

Numerous studies have confirmed that female sex hormones affect the cardiovascular system. Estrogen receptors are present on cells that line the interior surface of blood vessels, lymphatic vessels, and vascular smooth muscles. These receptors are involved in regulating the resistance of blood flow through the circulatory system [10]. Moreover, they may influence cardiovascular relaxation and contraction which may lead to the abnormal widening of neighboring blood vessels during vasovagal syncope [10]. Given the role that female sex hormones play in the cardiovascular system, it is probable that estrogen receptors also contribute to regulating the reflex responses which are responsible for the occurrence of vasovagal syncope. The incidence of vasovagal syncope is higher in women than in men and female sex hormones may be an important contributing factor [10]. This provides an important avenue for future investigation.

Symptoms of vasovagal syncope, including dizziness, nausea, and abdominal pain, can often be confused with menstrual symptoms experienced by women causing vasovagal syncope to be commonly misdiagnosed as symptoms of menstruation. Diagnostic methods should be improved to better distinguish symptoms of syncope from those accompanying menstruation.

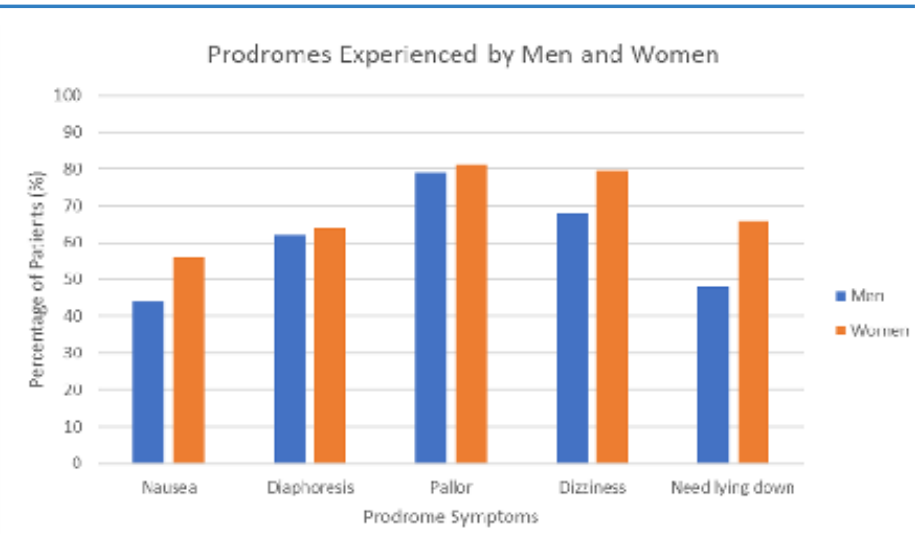

Figure 2. Prodromal symptoms of vasovagal syncope experienced by men and women. Figure produced with data from [7].

\section{IMPROVING DIAGNOSTIC CRITERIA FOR VASOVAGAL SYNCOPE}

To improve diagnosis and subsequent treatment of vasovagal syncope, it would be beneficial to collect more 
detailed personal and family history from patients. In one study, researchers administered a questionnaire to 418 patients diagnosed with syncope [11]. The questionnaire assessed a number of factors known to be related to syncope; the occurrence of each factor was compared between patients with positive tilt test results and those with syncope of other known causes. With this information, researchers were able to develop a specific set of questions to determine whether patients had vasovagal syncope. These questions and resulting score correctly classified $90 \%$ of patients, diagnosing vasovagal syncope with $89 \%-91 \%$ accuracy [11]. Although not completely accurate, this study shows the potential of using specific diagnostic criteria to obtain largely accurate diagnoses. Sheldon et al., provided an evidencebased method that can potentially be used to increase the correct diagnosis of vasovagal syncope. Since the ability to have a syncope expert in emergency hospital rooms at all times is impractical, questionnaire's such as these will allow doctors to diagnose vasovagal syncope correctly [12].

\section{CONCLUSION}

Although vasovagal syncope is a common condition, it often presents differently based on patients' age and sex. Vasovagal syncope is more common in younger age groups, especially among young women. Differing causes of syncope between age groups can also lead to differences in symptoms. For women, vasovagal reflex may present with some of the same symptoms as menstruation, leading to a failure to corectly diagnose the condition.

Failed or incorrect diagnoses and improper treatment have a significant impact on a patient's quality-of-life. With careful consideration of family and personal history, there is a greater chance of correct diagnosis. Medical professionals and researchers must improve their understanding of symptoms in patients of differing sex and ages to ensure a proper diagnosis. By taking a more comprehensive history of patients showing symptoms of vasovagal syncope, and considering their sex and age, medical professions should be able to provide more accurate diagnoses, and therefore, proper treatment as well.

\section{REFERENCES}

1. Benditt D, Kowey P, Downey B. Reflex syncope in adults: Clinical presentation and diagnostic evaluation. UpToDate [Internet]. 2017;1-23.
2. Petkar S, Cooper P, Fitzpatrick AP. How to avoid a misdiagnosis in patients presenting with transient loss of consciousness. Postgrad Med J. 2006;82(972):630-41.

3. Sun BC. Quality-of-Life, Health Service Use, and Costs Associated With Syncope. Prog Cardiovasc Dis [Internet]. 2013;55(4):370-5.

4. Alboni P, Furlan R. Vasovagal syncope. Vasovagal Syncope. 2015;(June 1999):1-326.

5. Campagna JA, Carter C. Clinical relevance of the BezoldJarisch reflex. Anesthesiology. 2003;98(5):1250-60.

6. Parry SW, Kenny RA. Tilt table testing in the diagnosis of unexplained syncope. QJM - Mon J Assoc Physicians. 1999;92(11):623-9.

7. Romme JJCM, Dekker LRC, Reitsma JB. Influence of age and gender on the occurrence and presentation of reflex syncope. 2008;127-33.

8. Ungar A, Mussi C, Del Rosso A, Noro G, Abete P, Ghirelli $L$, et al. Diagnosis and characteristics of syncope in older patients referred to geriatric departments. J Am Geriatr Soc. 2006;54(10):1531-6.

9. Wieling W, Ganzeboom KS, Saul JP. Reflex syncope in children and adolescents W Wieling. Heart. 2004;90(9):1094-100.

10. Pietrucha $A Z$, Jędrzejczyk-Spaho J, Konduracka $E$, Bzukała I, Krawczyk K, Kruszelnicka-Kwiatkowska O, et al. How does the estimated phase of menstrual cycle or menopause influence the prevalence of vasovagal syncope induced by head-up tilt test. Cardiol J. 2017;24(5):523-9.

11. Sheldon R, Rose $S$, Connolly S, Ritchie D, Koshman M Lou, Frenneaux M. Diagnostic criteria for vasovagal syncope based on a quantitative history. Eur Heart J. 2006;27(3):344-50.

12. Morillo CA. Evidence-based common sense: The role of clinical history for the diagnosis of vasovagal syncope. Eur Heart J. 2006;27(3):253-4. 